\title{
Utilization of a mobile medical van for delivering pediatric care in the bateys of the Dominican Republic
}

\author{
Heather L. Crouse • Charles G. Macias • \\ Andrea T. Cruz • Kim A. Wilson • Susan B. Torrey
}

Received: 3 December 2009 /Accepted: 31 May 2010/Published online: 21 August 2010

(C) Springer-Verlag London Ltd 2010

\begin{abstract}
Background Bateys are impoverished areas of housing for migrant Haitian sugar cane workers in the Dominican Republic (DR). In these regions, preventative health care is almost non-existent, public service accessibility is limited, and geographic isolation prevents utilization of care even by those families with resources. Consequently, the development of a viable mobile system is vital to the delivery of acute and preventative health care in this region. Aims This study evaluated an existing mobile medical system. The primary goal was to describe the population served, diseases treated, and resources utilized. A secondary goal was to determine qualitatively an optimal infrastructure for sustainable health care delivery within the bateys.

Methods Information on basic demographic data, diagnosis, chronicity of disease, and medications dispensed was collected on all pediatric patients seen in conjunction with an existing mobile medical system over a 3-month period in the DR. Health statistics for the region were collected and interviews were conducted with health care workers
\end{abstract}

The views expressed in this paper are those of the author(s) and not those of the editors, editorial board or publisher.

H. L. Crouse - C. G. Macias - A. T. Cruz • S. B. Torrey Department of Pediatrics, Section of Emergency Medicine, Baylor College of Medicine, Texas Children's Hospital, Houston, TX, USA

\section{K. A. Wilson}

Department of General Pediatrics, Harvard University,

Boston Children's Hospital,

Boston, MA, USA

\section{H. L. Crouse $(\square)$}

Texas Children's Hospital,

6621 Fannin St. MC 1-1481,

Houston, TX 77030-2399, USA

e-mail: hlcrouse@texaschildrenshospital.org
(HCWs) and community members on existing and optimal health care infrastructure.

Results Five hundred eighty-four pediatric patients were evaluated and treated. Median age was 5 years (range 2 weeks to 20 years), and $53.7 \%$ of patients seen were 5 years of age or younger. The mean number of complaints per patient was 2.8 (range 0 to 6). Thirty-six percent (373) of all diagnoses were for acute complaints, and 64\% (657) were chronic medical problems. The most common pediatric illnesses diagnosed clinically were gastrointestinal parasitic infection (56.6\%), skin/fungal infection (46.2\%), upper respiratory tract infections (URIs) (22.8\%), previously undiagnosed asthma and allergies (8.2\%), and symptomatic anemia (7.2\%). Thirty HCWs and community members were interviewed, and all cited the need for similar resources: a community clinic and hospital referral site, health promoters within each community, and the initiation of pediatric training for community HCWs.

Conclusion A mobile medical system is a sustainable, efficient mechanism for delivering acute and preventive care in the Haitian bateys of the Dominican Republic. The majority of patients served were 8 years of age or younger with multiple presenting symptoms. A pediatric protocol for identifying the most appropriate drugs and supplies for mobile units in the DR can be created based upon diseases evaluated. Qualitative data from HCWs and community members identified the need for an integrative health care delivery infrastructure and community health promoters versed in pediatric care who can aid in education of batey members and monitor chronic and acute illnesses. We are planning follow-up visits to implement these programs.

Keywords Mobile medical system $\cdot$ Pediatrics $\cdot$ Pediatric emergency medicine · Dominican Republic - International health $\cdot$ Developing country $\cdot$ Disease prevalence 


\section{Background}

Migrant Haitian sugar cane workers in the Dominican Republic (DR) typically live in impoverished areas of company housing referred to as bateys. Health care services are almost non-existent in these communities. Residents are legally, politically, socioeconomically, and geographically marginalized. Children born in the bateys lack birth registration and are unable to access public services including health care and, often, school. The estimated number of undocumented Haitians living in the bateys is between 650,000 to 1 million people. The bateys range in size from just a few to several thousand families and are located miles apart, with few communicating roads. Some bateys are remote and difficult to access by vehicle. During the rainy season, many are completely isolated, as the mud roads become impassable. This geographic isolation can prevent access to health care even for those families with legal rights. Consequently, developing a health care infrastructure that could provide mobile services for acute and preventative health care would be vital to these communities.

Limited information is available to guide the planning and implementation of mobile health units [1-7]. Factors to consider include the medical and social needs of a targeted population, the provider's resources, and the services that will be offered $[8,9]$. There are minimal baseline data describing health care availability or disease prevalence, particularly for pediatric patients, in remote communities. Consequently, it is difficult to design health care delivery options that will meet these poorly defined needs. Our goals were to describe the basic health care needs of children living in bateys in one region of the Dominican Republic and to suggest infrastructural changes that could optimize the delivery of health services to them.

\section{Methods}

Study design

This was a prospectively collected descriptive study.

Study setting and population

An area of approximately 32 bateys near San Pedro de Marcoris, DR, with an existing mobile medical system was chosen for this pilot project.

Study protocol

Pediatric patients were defined as 0-18 years old. Diagnoses were made clinically, since there were no laboratory services on the mobile medical van. Clinical diagnostic criteria were developed for several common diseases. These included parasitic infections, anemia, and atopy (see Table 1). Other diagnoses were made clinically according to the individual health care providers. All patient diagnoses and treatments were recorded.

Information describing the existing health care delivery system, perceived needs, and barriers was obtained through extensive open-ended interviews that were conducted with a convenience sample of 30 health care workers (HCWs) who worked in the bateys. Health statistics for the region were collected from the Ministry of Public Health. Data and information were collected as part of a quality improvement initiative to evaluate the program. The Institutional Review Board at Baylor College of Medicine approved the use of this data.

\section{Measurements}

Data were collected for each pediatric patient encounter and included patient demographics, presenting symptoms, diagnoses made, and medicines dispensed.

\section{Data analysis}

Data described above were collected and recorded on Teleforms and analyzed using Statistical Package for the Social Sciences, version 16.0 (SPSS; Chicago, IL). All patients evaluated were included in the data calculation. Statistical tests used to describe continuous variables included means and medians. Percentages were calculated for presenting symptoms, acute and chronic diagnoses, and medication utilization. Additionally, 95\% confidence intervals (CIs) were calculated using a binomial approximation of a normal distribution for acute and chronic diagnoses and medication utilization.

Table 1 Clinical criteria for diagnoses

\begin{tabular}{ll}
\hline Diagnosis & Clinical criteria \\
\hline Parasitic infection & $\begin{array}{c}\text { History of chronic abdominal pain with } \\
\text { diarrhea and/or severe abdominal pain, } \\
\text { or report of worms in stool } \\
\text { Complaint of weakness with associated } \\
\text { scleral icterus or conjunctival pallor, } \\
\text { nailbed pallor and tachycardia without } \\
\text { alternative explanation defined by } \\
\text { age-appropriate ranges } \\
\text { History of chronic cough and wheezing } \\
\text { at time of presentation } \\
\text { History of clear rhinnorhea and itching } \\
\text { or hives not associated with fever or } \\
\text { other skin infection }\end{array}$ \\
\hline
\end{tabular}


Table 2 Most common acute diagnoses

\begin{tabular}{lll}
\hline Diagnosis & \multicolumn{2}{l}{ Acute diagnoses made (total N=373) } \\
\cline { 2 - 3 } & Number & $\%(95 \% \mathrm{CI})$ \\
\hline URI & 133 & $35.6(30.9-40.6)$ \\
Headache & 56 & $15.0(11.8-19.0)$ \\
Skin superinfection & 47 & $12.6(9.6-16.4)$ \\
Fever & 30 & $8.0(5.7-11.3)$ \\
Ecthyma & 22 & $5.9(3.9-8.8)$ \\
Otitis media & 20 & $5.4(3.5-8.1)$ \\
Asthma exacerbation & 18 & $4.8(3.1-7.5)$ \\
Dehydration & 8 & $2.1(1.1-4.2)$ \\
Pharyngitis & 8 & $2.1(1.1-4.2)$ \\
UTI & 7 & $1.9(0.9-3.8)$ \\
Pneumonia & 4 & $1.1(0.4-2.7)$ \\
Other & 20 & $5.4(3.5-8.1)$ \\
\hline
\end{tabular}

\section{Results}

Between 1 February 2006 and 30 April 2006, 584 pediatric patients were evaluated and treated. Boys accounted for $50.1 \%$ of patients; mean age was 5.8 years with a median age of 5 years (range 2 weeks-20 years). Patient age was unavailable for 10 of 584 patients. Of the remaining 574 patients, $12.9 \%$ were under 1 year of age, $40.8 \%$ were $1-$ 5 years of age, $36.4 \%$ were $6-12$ years of age, and $9.9 \%$ were greater than 12 years of age, $72.3 \%$ were under 8 years of age, and $9.8 \%$ were over 12 years of age. One patient 20 years of age was evaluated along with her siblings, and the data were included in the results.

Of the 584 patients included, the most common presenting symptoms were abdominal pain $(52.9 \%)$, rash $(36.3 \%)$, decreased appetite (34.6\%), rhinorrhea (30.3\%), cough (22.1\%), and diarrhea (21.4\%). Most children had multiple

Table 3 Most common chronic diagnoses

\begin{tabular}{lll}
\hline Diagnosis & \multicolumn{2}{l}{ Chronic diagnoses made (total N=657) } \\
\cline { 2 - 3 } & Number & $\%(95 \% \mathrm{CI})$ \\
\hline Parasite/ameba/giardia & 331 & $50.4(46.6-54.2)$ \\
Scabies & 68 & $10.4(8.3-12.9)$ \\
Tinea capitus & 48 & $7.3(5.6-9.6)$ \\
Symptomatic anemia & 42 & $6.4(4.8-8.5)$ \\
Tinea corporis & 40 & $6.1(4.5-8.2)$ \\
Allergies & 30 & $4.6(3.2-6.4)$ \\
Dermatitis/eczema & 26 & $4.0(2.7-5.7)$ \\
Tinea versicolor & 17 & $2.6(1.6-4.1)$ \\
Failure to thrive & 8 & $1.2(0.6-2.4)$ \\
Other & 47 & $7.2(5.4-9.4)$ \\
\hline
\end{tabular}

complaints. The mean number of presenting complaints per patient was 2.8 (range 0 to 6). Only one child had a single chief complaint. Eighty-nine children (15.2\%) had two complaints, 198 (33.9\%) had three, 154 (26.3\%) had four, $103(17.7 \%)$ had five, and 39 (6.7\%) had $\geq$ six presenting complaints. Of all diagnoses made, 373 (36.2\%, 95\% CI 33.339.2) were acute, and 657 (63.8\%, 95\% CI 60.8-66.7) were chronic (Tables 2 and 3). The most common pediatric illnesses and medications dispensed are listed in Tables 4 and 5 .

Thirty HCWs and community members were interviewed with open-ended questions regarding the perceived health care needs of the children in the area and how to best meet these needs. A common response to interview questions was that the mobile van provides intermittent care, approximately one visit per batey per month, but there is no standardized referral system in place for a community clinic or hospital in case a patient needs a higher level care. In between mobile van visits, there is no mechanism to notify the van in case of an emergency. Many people noted that it would be helpful to have trained community health care workers in each batey who could serve as liaisons between the community and the van. These individuals would be grassroots advocates from the community who receive basic medical education and assist in educating the local population and identifying early illness. Nearly all

Table 4 Diagnoses

\begin{tabular}{llll}
\hline \multirow{2}{*}{ Category } & Diagnosis & \multicolumn{2}{l}{$\begin{array}{l}\text { Patients receiving diagnosis } \\
\text { (total N=584) }\end{array}$} \\
\cline { 3 - 4 } & & Number & $\%(95 \% \mathrm{CI})$ \\
\hline GI infections & Parasites & 319 & $54.6(50.5-58.6)$ \\
& Ameba & 10 & $1.7(0.9-3.1)$ \\
& Giardia & 2 & $0.3(0.1-1.2)$ \\
Skin infections & Scabies & 68 & $11.6(9.2-14.5)$ \\
& Skin superinfection & 47 & $8.0(6.1-10.5)$ \\
& Ecthyma & 22 & $3.8(2.5-5.6)$ \\
& Dermatitis/eczema & 26 & $4.5(3.1-6.4)$ \\
& Tinea capitis & 48 & $8.2(6.3-10.7)$ \\
& Tinea corporis & 40 & $6.8(5.1-9.2)$ \\
& Tinea versicolor & 17 & $2.9(1.8-4.6)$ \\
& Candida & 2 & $0.3(0.1-1.2)$ \\
ENT/pulmonary infections & URI & 133 & $22.8(19.6-26.4)$ \\
& Allergies & 30 & $5.1(3.6-7.2)$ \\
& Asthma & 18 & $3.1(2.0-4.8)$ \\
& Otitis media & 20 & $3.4(2.2-5.2)$ \\
& Symptomatic anemia & 42 & $7.2(5.4-9.6)$ \\
& Headache & 56 & $9.6(7.5-12.3)$ \\
& Fever & 29 & $5.0(3.5-7.0)$ \\
& Other & 188 & $32.2(28.5-36.1)$ \\
& & &
\end{tabular}


Table 5 Medications given ${ }^{\mathrm{a}}$

\begin{tabular}{|c|c|c|c|}
\hline \multirow[t]{2}{*}{ Category } & \multirow[t]{2}{*}{ Medication given } & \multicolumn{2}{|c|}{ Patients receiving medication (total $\mathrm{N}=584$ ) } \\
\hline & & Number & $\%(95 \% \mathrm{CI})$ \\
\hline \multirow[t]{3}{*}{ GI medications } & Mebendazole & 179 & $30.7(27.0-34.5)$ \\
\hline & Albendazole & 150 & $25.7(22.3-29.3)$ \\
\hline & Metronidazole & 8 & $1.4(0.7-2.7)$ \\
\hline \multirow[t]{6}{*}{ Skin medications } & Lindane & 57 & $9.8(7.6-12.4)$ \\
\hline & Permethrin & 8 & $1.4(0.7-2.6)$ \\
\hline & Vaseline & 11 & $1.9(1.1-3.3)$ \\
\hline & Griseofulvin & 29 & $5.0(3.5-7.0)$ \\
\hline & Antifungal cream & 53 & $9.1(7.0-11.7)$ \\
\hline & Steroid cream & 21 & $3.6(2.4-5.4)$ \\
\hline \multirow[t]{3}{*}{ Antibiotics } & Amoxicillin/clavunate & 29 & $5.0(3.5-7.0)$ \\
\hline & Amoxicillin & 42 & $7.2(5.4-9.6)$ \\
\hline & Trimethoprim-sulfamethoxazole & 10 & $1.7(0.9-3.1)$ \\
\hline \multirow[t]{5}{*}{ ENT/pulmonary medications } & Albuterol MDI & 5 & $0.9(0.4-2.0)$ \\
\hline & Albuterol liquid & 12 & $2.1(1.2-3.6)$ \\
\hline & Diphenhydramine & 22 & $3.8(2.5-5.6)$ \\
\hline & Cetirizine & 12 & $2.1(1.2-3.6)$ \\
\hline & Prednisone & 4 & $0.7(0.3-1.7)$ \\
\hline \multirow[t]{3}{*}{ Over-the-counter } & Acetaminophen & 80 & $13.7(11.2-16.7)$ \\
\hline & Ibuprofen & 58 & $9.9(7.8-12.6)$ \\
\hline & Cold medication $^{\mathrm{a}}$ & 81 & $13.9(11.3-16.9)$ \\
\hline \multirow[t]{2}{*}{ Vitamins } & Multivitamin & 362 & $62.0(58.0-65.8)$ \\
\hline & Iron & 32 & $5.5(3.9-7.6)$ \\
\hline Other & Other & 33 & $5.7(4.1-7.8)$ \\
\hline
\end{tabular}

${ }^{\mathrm{a}}$ This study was performed prior to the Food and Drug Adminstration warning against the use of cough suppressants in children $<2$ years of age

HCWs described feeling uncomfortable delivering pediatric care due to a lack of specialized training.

\section{Discussion}

This study demonstrated that a mobile medical van can provide a variety of important interventions for children living in the bateys who have limited access to medical services. These include the following:

- The majority of children visiting the mobile medical van had common illnesses that are simple to treat (rather than exotic conditions requiring specific medications), suggesting that health care workers traveling to resourcelimited settings should be familiar with both general pediatrics and local disease prevalence. As an example, the overall prevalence of atopic disease, subdivided into asthmatics and allergic patients, was $12.7 \%$. This is likely an underestimation, given the frequency of cough as a presenting symptom and that patients who received a diagnosis of asthma had wheezing at the time of encounter. Despite these strict clinical criteria, the number approaches the prevalence of atopic disease in American children (about 17\%) [10].

- Social marginalization and lack of access to health care may account for the large number of presenting complaints per patient (2.8) and the high percentage of chronic disease identified (63\%). A sustainable, reliable health care system could provide timely interventions for acute conditions and possibly decrease the incidence of exacerbations of chronic problems.

- Objective symptoms of anemia (likely iron deficiency) were present in $7 \%$ of children at the time of evaluation. Anemia can be associated with malnutrition and parasitic/helminthic diseases [11]. The majority of these children were clinically malnourished, and more than half were symptomatic from presumed parasitic infection. Several studies have implicated anemia and parasitic infections as causes of poor scholarly achievement [12, 13]. Periodic deworming (which is a safe, effective, and appropriate method for anemia prevention in the 
developing world) [14] may be an intervention that the mobile medical van could perform that would ameliorate the effects of anemia on the physical and cognitive development of children in the bateys.

- Vaccines can be administered during mobile medical van visits, since this is often the only source of health care for children living in the bateys. The Dominican government supplies free vaccines to the population as a whole; the issue then becomes vaccine delivery to rural areas. During the 3-month period of the study, a governmental public health nurse with a cooler of vaccines traveled with the mobile medical van once every 2 weeks. Most families receiving vaccinations carefully maintained the immunization records of their children and were grateful to have catch-up vaccines available on the van. It would be optimal to have immunizations available on all community visits to the bateys, and measures are being taken to facilitate and improve the relationship between the mobile medical van and government-run public health clinics.

Several discrepancies exist between the prevalence of diagnoses listed and medications dispensed. This may be because treatment options were often contingent upon availability of medications. Many medication shortages were encountered in the "travel med pack" options donated from companies in the United States. Though generous, the packs often did not include medicines to treat parasites, scabies, or fungal infections, some of the most prevalent diseases seen in the DR. Consequently, when stocking a local mobile medical unit in the DR or for mobile teams from the US and abroad, donated medicine packs should be modified to reflect the needs of the population.

There are several limitations to this study. Though adults were simultaneously evaluated and treated by the general physician on the mobile medical van, only data from pediatric patients evaluated by the US medical team were recorded, precluding estimates of relative need for adult and pediatric medications in mobile medical systems. Nearly all diagnoses made were based on clinical findings and definitions. Very few diagnoses were made based on laboratory findings; in those instances, patients presented to the mobile medical van with laboratory results from tests performed elsewhere. Since the mobile van visits the bateys during the week (Monday through Friday) and always during the day, the relative paucity of older children in our study could represent sampling bias, since older children may have been at school or working in the fields at the time of our visits. Road conditions can severely limit access to more remote bateys, especially during the rainy season. Consequently, the generalizability of this study could be limited to the dry season or to bateys closer to major roads.
With knowledge of the most common chief complaints and diagnoses obtained from this pilot study, it will be easier to stock a mobile medical unit with communityspecific supplies. Additionally, pediatric protocols can be developed for use by medical personnel from the mobile medical van and other local generalist physicians, nurses, and community health workers. This information could also be used to develop a pediatric HCW curriculum that would be generalizeable to other bateys in the DR and possibly other remote rural areas in the developing world. In addition to the treatment of acute disease, chronic illnesses could also be tracked and treated; these could include asthma, parasitic diseases (e.g., periodic deworming of pediatric patients), tuberculosis (TB), and HIV screening. Finally, the laboratory capacity of the medical van could be augmented to include basic screening tests for conditions that occur commonly, such as a glucometer (diabetes), rapid hemoglobin testing (anemia), urine dipsticks (urinary tract infections), and tuberculin skin test placement. We are planning follow-up visits to implement these programs.

The DR-based mobile medical team and the government are currently discussing the development of several public health referral clinics within the bateys. They are in the primary planning stages of augmenting the capabilities of the mobile medical van by creating a more integrative health care system involving a referral hospital and liaison community health workers.

\section{Conclusions}

A mobile medical system is a practical mechanism for delivering acute and preventive care to the Haitian bateys in the Dominican Republic. The majority of patients served are 5 years of age or younger, with multiple presenting symptoms. A pediatric protocol for identifying the most appropriate drugs and supplies for the mobile unit or for mobile teams from the United States and other countries can be developed based upon the diseases that are prevalent in the region. Rigorous preparation can decrease cost and increase the effectiveness of donated medicines and supplies.

Qualitative data from health care workers and community members identified a need for an integrated health care delivery infrastructure that would link the mobile medical van to a referral clinic or hospital as well as for community health promoters who can aid in education of batey members and monitoring of chronic and acute illnesses. The initiation of pediatric training for community workers is also warranted.

A mobile medical system has the potential for both short-term and long-term interventions. Feasible short-term 
interventions by traveling medical teams include diagnosis and treatment of acute conditions, periodic deworming, catch-up vaccines and short-term treatment of anemia. Long-term sustainability is the goal of a mobile unit run by the home country where a true health care infrastructure can be developed, acute illnesses can be treated, chronic illnesses monitored, immunization programs implemented, and community members educated.

Acknowledgments I would like to thank the following people for their help in collecting and organizing the above data: Sister Concepción Rivera, RN; Chantal Caviness, MD, MPH, PhD; Troy Bush, BS-CCRP; Jennifer Jones, MS; Deborah Hsu, MD, MEd; Eitan Rubinstein, MD; Christiana Russ, MD; Rachel Thompson, MD; and Emily Willner, MD.

\section{Conflicts of interest None.}

\section{References}

1. Alexy B, Elnitsky C (1996) Community outreach: rural mobile health unit. J Nurs Admin 26(12):38-42

2. Dyer J (1996) Comparative costs of mobile and fixed-clinic primary health care services. S Afr Med J 86(5):528-530

3. Fox-Rush JA, Foord F (1996) Costs, effects and cost-effectiveness analysis of a mobile maternal health care service in West Kiang. Health Policy 32(2):123-143

4. Garrett D (1995) Mobile access: opening health care doors. Nurse Manager 26(10):29

5. Geller JL, Fisher WH, McDermeit M (1995) A national survey of mobile crisis services and their evaluation. Psychiatr Serv 46 (9):893-897

6. Hewitt S, Jarrett L, Winter B (1996) Emergency medicine at a large rock festival. J Accid Emerg Med 13(1):26-27

7. McNeal G (1966) Mobile health care for those at risk. Health Care Perspect Community 17(3):134-140

8. Muolavi D, Bushy A, Peterson J, Stullenbarger B (1999) Factors to consider when purchasing a mobile health unit to deliver services. J Nurs Admin 29(2):34-41

9. Muolavi D, Bushy A, Peterson J, Stullenbarger B (2000) Thinking about a mobile health unit to deliver services? Things to consider before buying. Austr J Rural Health 8(6):8-16

10. Hanifin JM, Reed ML, Eczema Prevalence and Impact Working Group (2007) A population-based survey of eczema prevalence in the United States. Dermatitis 18(2):82-91

11. Ezeamama AE, Friedman JF, Olveda RM, Acosta LP, Kurtis JD, Mor V, McGarvey ST (2005) Functional significance of lowintensity polyparasite helminth infections in anemia. J Infect Dis 192(12):2160-2170

12. Ezeamama AE, Friedman JF, Acosta LP, Bellinger DC, Langdon GC, Manalo DL, Olveda RM (2005) Helminth infection and cognitive impairment among Filipino children. Am J Trop Med Hyg 72(5):540-548

13. Hutchinson SE, Powell CA, Walker SP, Chang SM, GranthamMcGregor SM (1997) Nutrition, anaemia, geohelminth infection, and school achievement in rural Jamaican primary school children. Eur J Clin Nutr 51(11):729-735

14. Mohapatra SC, Gaur SD (1996) Effect of periodic deworming on haemoglobin level of a rural community in Varanasi. Ind J Prevent Soc Med 27(1\&2):17-22

Heather Crouse, MD completed medical school at the University of North Carolina in Chapel Hill, NC. She then went on to complete residency in the Boston Combined Pediatric Residency at Children's Hospital Boston and Boston Medical Center in 2005. She completed a combined 4-year subspecialty fellowship in Pediatric Emergency Medicine and International Health at Baylor College of Medicine and Texas Children's Hospital, and has recently joined the faculty there as Assistant Professor of Pediatrics.

Charles G. Macias MD, MPH is a graduate of Stanford University, Southwestern Medical School and the UT School of Public Health, Houston. He is board certified in Pediatrics and Pediatric Emergency Medicine and is an Associate Professor of Pediatrics at Baylor College of Medicine. He is a founding member of the Pediatric Emergency Research Network, a global research network comprised of 6 independent pediatric emergency medicine research networks.

Andrea Cruz, MD, MPH completed undergraduate training at Harvard University in Cambridge, MA and medical school at Vanderbilt University in Nashville, TN. She then completed pediatric residency and combined fellowships in pediatric emergency medicine and infectious diseases at Baylor College of Medicine in Houston, Texas. She graduated from the University of Texas at Houston School of Public Health with a masters of public health concentrating on epidemiology and global health. She has recently joined the faculty at Baylor College of Medicine as an assistant professor of pediatrics.

Kim Wilson, MD, MPH is an assistant professor at Harvard Medical School and the Associate Director for the Center for Global Pediatrics at Children's Hospital Boston. Dr. Wilson completed her MD and MPH at Harvard Medical School and Harvard School of Public Health, and completed a residency in pediatrics and a general academic pediatric fellowship at Children's Hospital Boston. Dr. Wilson's professional focus has been on improving the quality of child health care for under-served populations domestically and internationally.

Susan B. Torrey, MD completed medical school at the University of Connecticut. She was a resident in pediatrics at St. Christopher's Hospital for Children in Philadelphia. She completed her pediatric emergency medicine fellowship at Children's Hospital of Philadelphia. She has been an associate professor of pediatrics at Baylor College of Medicine since 2007, where she is the director of the global health track in the pediatric emergency medicine fellowship program. 